\title{
Involvement of GJA1 and Gap Junctional Intercellular Communication between Cumulus Cells and Oocytes from Women with PCOS
}

\author{
Qiwei Liu $\mathbb{D}^{1},{ }^{1}$ Liang Kong, ${ }^{1}$ Junhui Zhang, ${ }^{2}$ Qian Xu, ${ }^{1}$ Jingxue Wang $\mathbb{D},{ }^{1}$ Zhigang Xue $\mathbb{D},{ }^{3}$ \\ and Jinjuan Wang $\mathbb{D}^{1}$ \\ ${ }^{1}$ Department of Gynecological Minimal Invasive Center, Beijing Obstetrics and Gynecology Hospital, Capital Medical University, \\ Beijing 100010, China \\ ${ }^{2}$ Anhui Province Key Laboratory of Reproductive Health and Genetics, Anhui Medical University, Hefei, 230032 Anhui, China \\ ${ }^{3}$ Translational Center for Stem Cell Research, Tongji Hospital, Department of Regenerative Medicine, Tongji University School \\ of Medicine, Shanghai, China
}

Correspondence should be addressed to Zhigang Xue; xuezg@tongji.edu.cn and Jinjuan Wang; wangjinjuan2007@sina.com

Received 24 December 2019; Accepted 14 February 2020; Published 29 February 2020

Academic Editor: Enrique Gomez

Copyright $\odot 2020$ Qiwei Liu et al. This is an open access article distributed under the Creative Commons Attribution License, which permits unrestricted use, distribution, and reproduction in any medium, provided the original work is properly cited.

Polycystic ovary syndrome (PCOS) is a common female endocrine system disease that affects $17.8 \%$ of women of reproductive age and leads to infertility, obesity, glucose metabolic disorders, cardiovascular disease, and body-mind problems. However, the etiology of PCOS remains unclear. Follicular growth is disrupted as a result of ovarian hyperandrogenism and distorted intraovarian paracrine signaling in women with PCOS. Microcommunication between oocytes and cumulus cells plays a critical role in folliculogenesis. Gap junction alpha 1 (GJA1) plays a crucial role in the developing follicles by forming communication channels between cumulus cells and oocytes, but this has not yet been reported in women with PCOS. Therefore, we aimed to study the role of GJA1 in the microcommunication between oocytes and cumulus cells in women with PCOS. In our study, cumulus cell-oocyte complexes (COCs) from women were isolated via ultrasound-guided vaginal puncture, and oocytes were selected from COCs and categorized based on 3 oocyte maturation stages. Then, RT-qPCR and immunofluorescence analysis were performed to detect both the gene expression and protein of GJA1 in oocytes from women with and without PCOS. There was no statistically significant difference in age and BMI (body mass index), but patients with PCOS had a higher ratio of basic LH/FSH (luteinizing hormone/follicle-stimulating hormone), androstenedione, and total ovarian volume. The qRT-PCR results showed higher gene expression of GJA1 in oocytes without PCOS at the germinal vesicle (GV) stage compared with that of oocytes from women with PCOS. Immunofluorescence analysis showed that the expression level of GJA1 in oocytes from women with PCOS was very weak compared with that of oocytes from women without PCOS. In conclusion, GJA1 may play a critical role in the development of oogenesis arrest in women with PCOS throughout the oogenesis processes, including oogenesis and oocyte maturation.

\section{Introduction}

Polycystic ovary syndrome (PCOS), first identified in 1935 by Stein and Leventhal, is a female endocrine disorder characterized by hyperandrogenism, ovulatory dysfunction, and polycystic ovarian morphology that affects $17.8 \%$ of women of reproductive age [1-3]. PCOS causes a series of major sys- temic complications, including infertility, obesity, glucose metabolic disorders, cardiovascular disease, and body-mind problems, which impair female health [4-6]. Among these complications, infertility is one of the most challenging problems for reproductive women, accounting for $80 \%$ of anovulatory infertility cases in women with PCOS [7]. The etiology of PCOS remains unclear. Follicular growth is disrupted as a 
TABLe 1: Clinical characteristics and outcome of patients with and without PCOS.

\begin{tabular}{lccc}
\hline & Non-PCOS $(n=8)$ & PCOS $(n=8)$ & $P$ value \\
\hline Age (year) & $26.4 \pm 1.1$ & $25.9 \pm 0.8$ & 0.7035 \\
BMI $\left(\mathrm{kg} / \mathrm{m}^{2}\right)$ & $23.6 \pm 0.1$ & $23.1 \pm 1.7$ & 0.7804 \\
AFC & $7.8 \pm 0.5$ & $>24.000$ & $<0.001(0.000)$ \\
FSH $(\mathrm{mIU} / \mathrm{mL})$ & $7.4 \pm 0.7$ & $7.6 \pm 0.3$ & 0.7820 \\
LH (mIU/mL) & $5.9 \pm 0.8$ & $13.4 \pm 1.3$ & $<0.001(0.000)$ \\
LH/FSH & $3.5 \pm 0.5$ & $6.8 \pm 0.6$ & $<0.001(0.000)$ \\
Insulin (IU) & $12.4 \pm 1.7$ & $15.4 \pm 4.4$ & 0.8541 \\
Testosterone (nmol/L) & $0.9 \pm 0.1$ & $1.9 \pm 0.2$ & $<0.001(0.000)$ \\
Androstenedione (nmol/L) & $1.8 \pm 0.1$ & $2.3 \pm 0.3$ & 0.1611 \\
SHBG (nmol/L) & $54.2 \pm 7.0$ & $40.0 \pm 4.8$ & 0.1177 \\
\hline
\end{tabular}

BMI: body mass index; FSH: follicle-stimulating hormone; LH: luteinizing hormone, AFC: antral follicle; SHBG: sex hormone-binding globulin.

result of ovarian hyperandrogenism and distorted intraovarian paracrine signaling in women with PCOS, which makes follicle arrest at secondary follicle stage, and the size of follicles stays in the range of $2-9 \mathrm{~mm}$ [8]. The follicular microenvironment plays a crucial role in folliculogenesis. During folliculogenesis, oocytes receive important signaling and nutrition only through communication with the cumulus cells around them. Through this communication, oocytes and cumulus cells complete folliculogenesis, oocyte maturation, and ovulation [9-12]. Therefore, microcommunication plays a critical role in oocyte maturation.

Gap junctional intercellular communication (GJIC) has been reported to participate in many normal physiological and pathological processes. Gap junctions (GJs) are essential channels linking adjacent cells and allow for the exchange of molecules, nutrients, and signaling molecules between cells $[13,14]$. GJs encoded by connexins (Cxs) are a family of approximately 20 proteins that form gap junction channels that allow intercellular communication $[15,16]$. GJA1, also called connexin 43 (Cx 43), was documented to play a crucial role in forming communication channels that couple developing follicles to cumulus cells, assisting in communication between cumulus cells and oocytes. Studies have reported that folliculogenesis was arrested when GJA1 was knocked out in the mouse ovary, and the follicles stayed in the primary stage and developed incompetent oocytes [17]. And inhibiting expression level of GJA1 in granulosa cells in rats can cause inhibition of progesterone production [18]. With GJA1, oocytes obtain nutrients, ions, and signals from somatic cells around them to support oocyte maturation, regulate $\mathrm{pH}$, and maintain meiotic arrest $[19,20]$.

However, no studies have identified a role for GJA1 in oocytes and cumulus cells in follicles of women with PCOS. Our previous study showed that GJA1 was significantly differentially expressed between human oocytes with without PCOS [21]. In our study, we found that GJA1 expression was lower in PCOS oocytes at the GV stage than in healthy oocytes through single-cell RNA sequencing. To investigate the importance of GJA1 in the folliculogenesis arrest in PCOS, we further studied GJA1 in oocytes from women with PCOS.

\section{Materials and Methods}

2.1. Study Population. We recruited 16 women, including 8 women with PCOS and 8 without PCOS. All participants were undergoing assisted reproductive technology (ART) at Anhui First People's Hospital in Anhui, China, and were willing to donate oocytes. The study protocol was approved by the Research Ethics Committee of Anhui First People's Hospital (No. 2014008) and conducted in accordance with approved institutional guidelines. All participants gave written informed consent. PCOS patients were confirmed to have at least two of the three Rotterdam 2003 criteria for diagnosing PCOS: hyperandrogenism, oligoovulation and/or anovulation, and polycystic ovaries [22]. We excluded patients with Cushing's syndrome, congenital adrenal hyperplasia, and androgen-secreting tumors. Participant demographic and clinical characteristics, such as age, BMI, and LH, FSH, estradiol (E2), and progesterone levels, were recorded (Table 1).

2.2. Isolation of Oocytes. Ovarian stimulation for all participants was carried out according to the standard protocol. Cumulus cell-oocyte complexes (COCs) were isolated via ultrasound-guided vaginal puncture and washed in phosphate-buffered saline (PBS). Oocytes were selected from COCs and categorized based on the oocyte maturation stage: GV (oocyte maturation arrested in the prophase of meiosis), MI (metaphase I, first meiotic metaphase), and MII (metaphase II, second meiotic metaphase). Oocytes were prepared for use in RNA-seq experiments, and the remaining samples were prepared for quantitative reverse-transcriptase polymerase chain reaction (qRT-PCR) and immunofluorescence.

2.3. RNA Extraction and Real-Time PCR. RNA was extracted from single oocytes and converted into cDNA according to the Smart-seq2 protocol [23]. RNA was isolated from single oocytes using oligo-Dt30VN primers and dNTPs at $72^{\circ} \mathrm{C}$ and then reverse transcribed into cDNA. After the firststrand reaction, cDNA was amplified and purified twice by using AMPure XP beads and $80 \%$ ethyl alcohol. Significantly differentially expressed genes were confirmed by qRT-PCR 
TABle 2: Primers used for qRT-PCR.

\begin{tabular}{lcc}
\hline cDNA & Sense primer $\left(5^{\prime}-3^{\prime}\right)$ & Antisense primer $\left(5^{\prime}-3^{\prime}\right)$ \\
\hline GJA1 & CAATCTCTCATGTGCGCTTCT & GGCAACCTTGAGTTCTTCCTCT \\
GAPDH & ACCCGCCCTATCTCAACTACC & AGGACACCATAATGACAGCCT \\
\hline
\end{tabular}

GJA1: Gap junction alpha 1 protein; GAPDH: glyceraldehyde-3-phosphate dehydrogenase.

(quantitative reverse-transcriptase polymerase chain reaction) (208054, QIAGEN, Germany).

The sequences of the primers for GJA1 and glyceraldehyde-3-phosphate dehydrogenase (GAPDH) used in this study are shown in Table 2. GAPDH was used to normalize the gene expression of GJA1. qRT-PCR was performed in a total reaction volume of $25 \mu \mathrm{L}$, including $1 \mu \mathrm{L}$ cDNA $(1 \mathrm{ng} / \mu \mathrm{L}), 10 \mu \mathrm{L} 2 \mathrm{x}$ SYBR green PCR master mix, $0.1 \mu \mathrm{L}$ QN ROX reference dye, $1 \mu \mathrm{L}$ forward primer $(10 \mathrm{mmol} / \mathrm{L}), 1 \mu \mathrm{L}$ reverse primer $(10 \mathrm{mmol} / \mathrm{L})$, and $6.9 \mu \mathrm{L}$ RNase-free water. The PCR initial activation was achieved by heating the samples to $95^{\circ} \mathrm{C}$ for $2 \mathrm{~min}$, followed by a total of 40 cycles of denaturation at $95^{\circ} \mathrm{C}(5 \mathrm{~s})$ and $60^{\circ} \mathrm{C}(30 \mathrm{~s})$.

2.4. Immunofluorescence. Oocytes that were extracted from COCs were washed with PBS three times and then fixed for $10 \mathrm{~min}$ at room temperature in $4 \%$ paraformaldehyde. The oocytes were washed in $0.5 \%$ Triton- 100 three times and treated with $0.5 \%$ Triton X-100 at room temperature for $40 \mathrm{~min}$. Then, the oocytes were washed in 3\% normal goat serum three times and blocked with $3 \%$ normal goat serum for $60 \mathrm{~min}$. After that, the oocytes were washed once and then incubated with primary antibody (1:50 dilution, mouse antiGJA1 monoclonal antibody) at $4{ }^{\circ} \mathrm{C}$ overnight.

Then, the oocytes were washed with 3\% normal goat serum three times. Subsequently, the oocytes were washed once and then incubated with goat anti-mouse immunoglobulin $\mathrm{G}$ fluorescein second antibody ( $1: 100$ dilution) for $1 \mathrm{~h}$ at room temperature. The oocytes were washed with $3 \%$ normal goat serum three times. After washing, the DNA in the cells was labeled with propidium iodide (PI) (1:2000 dilution) for $10 \mathrm{~min}$. Finally, the oocytes were placed in a drop of $3 \%$ normal goat serum and observed under a fluorescence microscope.

2.5. Gene Expression Analysis and Statistics. Differential gene expression was considered significant if the adjusted $P($ Padj $)<0.05$. Padj was calculated by using the Benjamini-Hochberg method to exclude false-positive results. All clinical parameters are expressed as the mean \pm standard deviation depending on the distribution.

\section{Results}

3.1. Clinical and Biochemical Characteristics of Patients. The clinical characteristics of the 16 participants were analyzed and are shown in Table 1. There was no statistically significant difference in age or BMI, but patients with PCOS had increased LH $(13.4 \pm 1.3)$, ratio of basic LH/FSH $(6.8 \pm 0.6)$, androstenedione and total ovarian volume.
TABLE 3: Numbers of oocyte at different stages analyzed by qRTRNA and immunofluorescence.

\begin{tabular}{lcccc}
\hline & GV & MI & MII & Immunofluorescence \\
\hline Normal & 6 & 6 & 4 & 5 \\
PCOS & 6 & 7 & 4 & 5 \\
\hline
\end{tabular}

GV: germinal vesicle, oocyte maturation arrested in the prophase of meiosis; MI: metaphase I, first meiotic metaphase; MII: metaphase II, second meiotic metaphase.

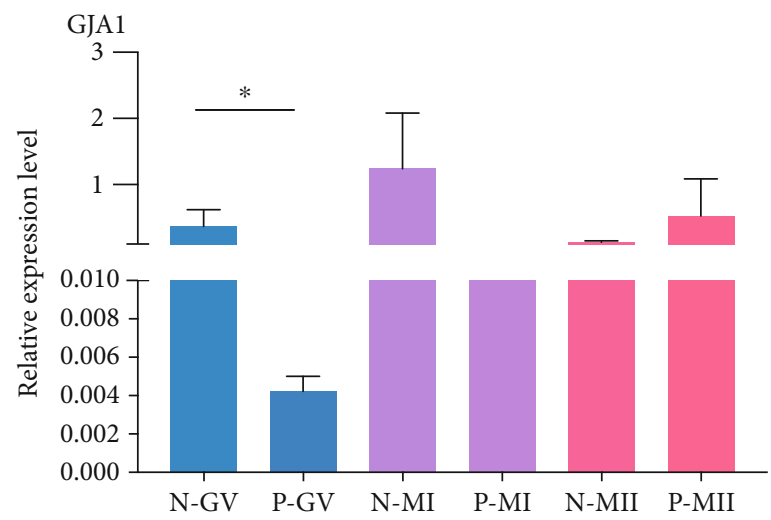

FIgure 1: Differentially expressed genes in oocytes from PCOS compared with oocyte from non-PCOS by RT-PCR. N-GV: gene expression levels in oocyte at GV stage from normal women; P-GV: gene expression levels in oocyte at GV stage from PCOS women; N-MI: gene expression levels in oocyte at MI stage from normal women; P-MI: gene expression levels in oocyte at MI stage from PCOS women; N-MII: gene expression levels in oocyte at MII stage from normal women; P-MII: gene expression levels in oocyte at MII stage from PCOS women. ${ }^{*} P$ value $<0.05$.

3.2. qRT-PCR Analysis. We randomly selected 43 oocytes, which were divided into the GV, MI, and MII groups according to the oocyte maturation stage (Table 3). The qRT-PCR results showed higher gene expression of GJA1 in oocytes without PCOS at the GV stage compared with that of oocytes with PCOS, which corresponded to the results of single-cell RNA-seq in a previous article (Figure 1). However, there were no significant differences in gene expression at the MII stages between oocytes with or without PCOS.

3.3. Immunofluorescence Analysis. To detect the expression pattern of GJA1 in oocytes, immunofluorescence analysis was performed (Figure 2). The results showed a positive fluorescence signal for GJA1 in oocytes both with and without PCOS at stage GV. Moreover, the expression level of GJA1 in oocytes from women with PCOS was much weaker compared with that of oocytes from women without PCOS. 


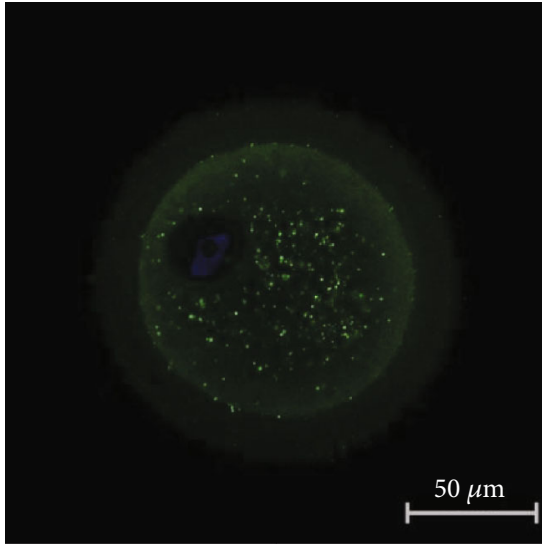

(a)

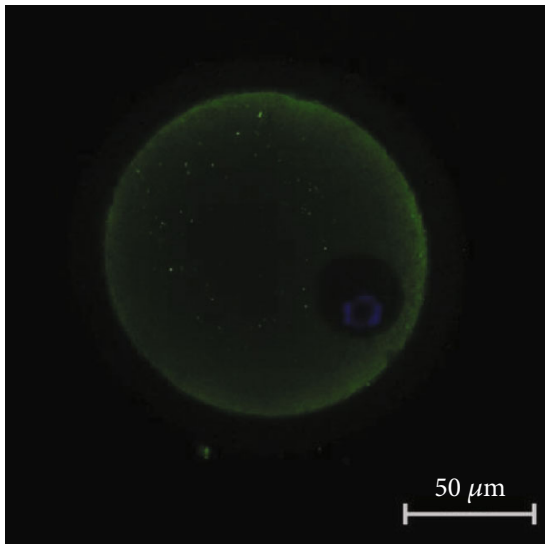

(d)

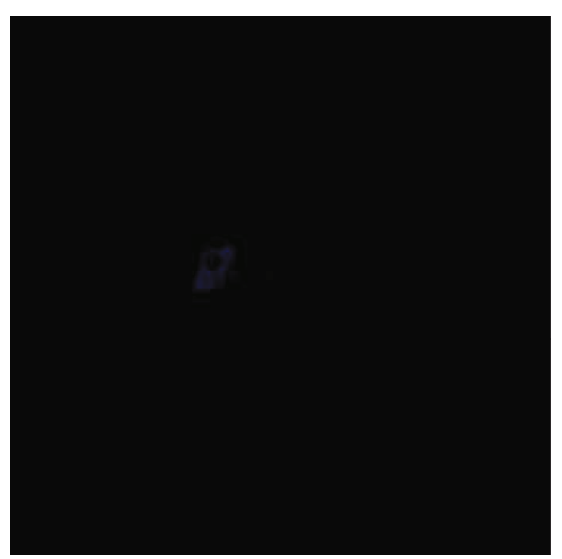

(b)

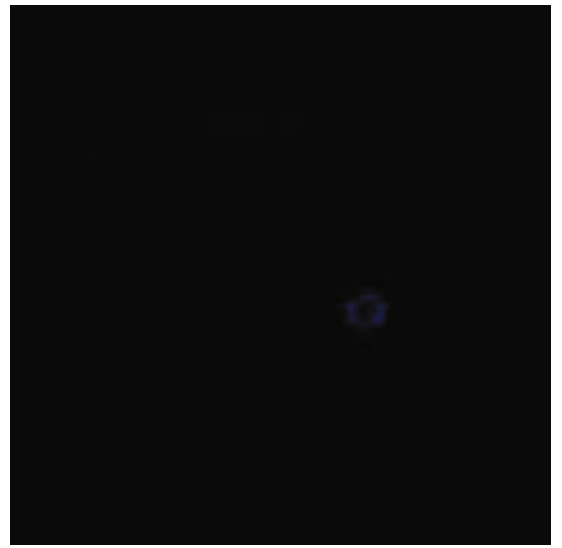

(e)

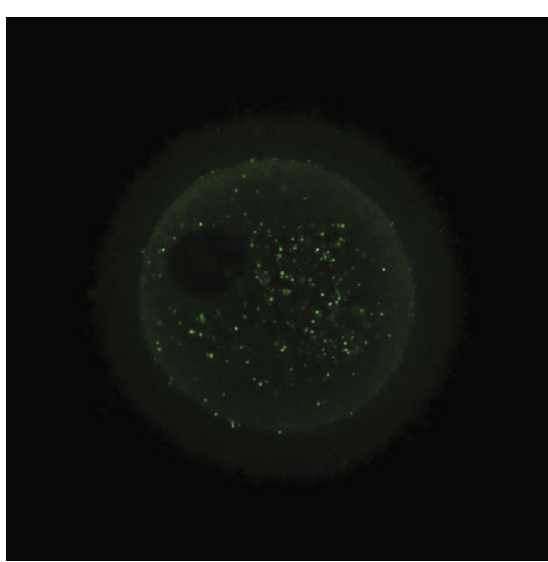

(c)

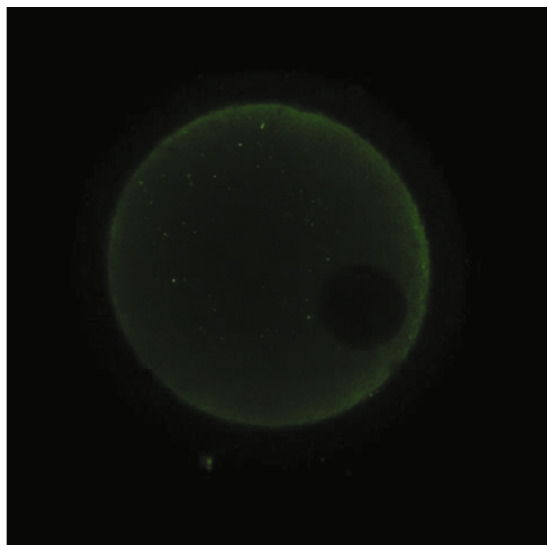

(f)

FIGURE 2: Immunofluorescence staining for detecting the expression pattern of GJA1 in human oocyte with and without PCOS at GV stage. $(a-c)$ Oocyte from women without PCOS. (d-f) Oocyte from PCOS women. Oocytes were counterstained with the cytoskeleton (green) and nuclei (DAPI, blue). Bar $=50 \mu \mathrm{m}$.

\section{Discussion}

In this study, we found that GJA1 was negatively expressed at both the gene and protein levels in oocytes with PCOS compared with non-PCOS oocytes, which was consistent with the outcomes of our previous study. These data suggest that GJA1 downregulation may be involved in folliculogenesis arrest in women with PCOS, which causes anovulatory infertility in these women. Androstenedione may play a role in the expression level of GJA1 both in rat polycystic ovary [18]. Our study also suggested that the gene expression of GJA1 was not different between oocytes from women with or without PCOS at the late stage of oogenesis after ART. This result indicates that ART may improve maturation capacity by regulating gap junctions.

Oocytes in follicles are tightly surrounded by cumulus cells and cannot directly obtain signals and nutrition from the microenvironment of the ovary. Therefore, cellular interaction between oocytes and somatic cells plays an indispensable role in oocyte growth and maturation and folliculogenesis [24-26]. One study showed that mice with disrupted GJs failed to undergo oocyte meiotic maturation and were infertile [17]. Gap junction intercellular interactions are maintained between somatic cells and oocytes during the early stage of oogenesis and are blocked by GJA1 phosphorylation when oocyte meiosis begins as the last stage [27, 28]. GJs provide nucleotides, amino acids, and energy substrates that the growing oocyte is unable to obtain itself from neighboring cells [29]. Several studies have suggested that deletion of Gja1 in granulose cells of mice prevents oocyte development $[17,30]$. In addition, cumulus cells also help to maintain a stable intracellular $\mathrm{pH}$ in oocytes through gap junction channels, which is a fundamental homeostatic process for the survival and proliferation of oocytes [31]. Communication with granulosa cells also promotes chromatin structure remodeling during the final phase of bovine oocyte differentiation and acquisition of meiotic competence [32].

At the last stage of folliculogenesis, MAPK is stimulated by $\mathrm{LH}$ through the ERK-MAPK signaling pathway and promotes GJA1 phosphorylation [33]. With GJA1 phosphorylation, the gap junction is lost, which decreases the concentration of cGMP (cyclic guanosine monophosphate) in oocytes and promotes the process of meiosis [34-36]. cAMP (cyclic adenosine monophosphate), a second messenger, has been known to induce oocyte meiotic processes. cGMP inhibits the hydrolysis of cAMP in oocytes, and so a low concentration of CGMP induces the hydrolysis of cAMP, which promotes the meiotic process and oocyte maturation. 
In conclusion, GJA1 may play a critical role in the development of oogenesis arrest in women with PCOS through all of the oogenesis processes, including oogenesis and oocyte maturation. Our study may provide a clue to determining the etiology of PCOS.

\section{Data Availability}

The data used to support the findings of this study are included within the article.

\section{Conflicts of Interest}

The authors declare that they have no competing interest.

\section{Authors' Contributions}

XZG and WJJ designed the study and analyzed the data from RNA-seq. LQW and ZJH performed the RT-PCR and immunofluorescence analysis. LQW, ZJH, and KL collected the COC samples. XQ and WJX organized the clinical data of participants. LQW and ZJH made the charts. All authors read and approved the final manuscript. Qiwei Liu and Liang Kong contributed equally to this work.

\section{Acknowledgments}

This study was supported by a grant from Beijing Obstetrics and Gynecology Hospital, Capital Medical University (FCYY201813).

\section{References}

[1] I. F. Stein and M. L. Leventhal, "Amenorrhea associated with bilateral polycystic ovaries," American Journal of Obstetrics and Gynecology, vol. 29, no. 2, pp. 181-191, 1935.

[2] A. Hosseinkhani, N. Asadi, M. Pasalar, and M. M. Zarshenas, "Traditional Persian medicine and management of metabolic dysfunction in polycystic ovary syndrome," Journal of Traditional and Complementary Medicine, vol. 8, no. 1, pp. 17-23, 2018.

[3] U. Branavan, K. Muneeswaran, W. S. S. Wijesundera, A. Senanayake, N. V. Chandrasekharan, and C. N. Wijeyaratne, "Association of Kiss1 and GPR54 gene polymorphisms with polycystic ovary syndrome among Sri Lankan women," BioMed Research International, vol. 2019, Article ID 6235680, 2019.

[4] M. Çetin, P. Tunçdemir, K. Karaman et al., "Cardiovascular evaluation and serum paraoxonase- 1 levels in adolescents with polycystic ovary syndrome," Journal of Obstetrics and Gynaecology, vol. 40, no. 1, pp. 90-95, 2019.

[5] A. M. Moore and R. E. Campbell, "Polycystic ovary syndrome: understanding the role of the brain," Frontiers in Neuroendocrinology, vol. 46, pp. 1-14, 2017.

[6] S. Tantanavipas, O. Vallibhakara, A. Sobhonslidsuk et al., "Abdominal obesity as a predictive factor of nonalcoholic fatty liver disease assessed by ultrasonography and transient elastography in polycystic ovary syndrome and healthy women," BioMed Research International, vol. 2019, Article ID 9047324, 2019.
[7] A. H. Balen, L. C. Morley, M. Misso et al., "The management of anovulatory infertility in women with polycystic ovary syndrome: an analysis of the evidence to support the development of global WHO guidance," Human Reproduction Update, vol. 22, no. 6, pp. 687-708, 2016.

[8] P. Pigny, E. Merlen, Y. Robert et al., "Elevated serum level of anti-mullerian hormone in patients with polycystic ovary syndrome: relationship to the ovarian follicle excess and to the follicular arrest," The Journal of Clinical Endocrinology and Metabolism, vol. 88, no. 12, pp. 5957-5962, 2003.

[9] S. Jonard and D. Dewailly, "The follicular excess in polycystic ovaries, due to intra-ovarian hyperandrogenism, may be the main culprit for the follicular arrest," Human Reproduction Update, vol. 10, no. 2, pp. 107-117, 2004.

[10] D. Das and S. Arur, "Conserved insulin signaling in the regulation of oocyte growth, development, and maturation," Molecular Reproduction and Development, vol. 84, no. 6, pp. 444-459, 2017.

[11] D. Panidis, K. Tziomalos, E. Papadakis, E. Kandaraki, and I. Katsikis, "The guidelines issued by the European Society for Human Reproduction and Embryology and the American Society for Reproductive Medicine regarding the induction of ovulation with metformin in patients with the polycystic ovary syndrome potentially require reconsideration," Hormones, vol. 12, no. 2, pp. 192-200, 2013.

[12] B. Chermula, M. Brazert, D. Izycki et al., "New gene markers of angiogenesis and blood vessels development in porcine ovarian granulosa cells during short-term primary culture in vitro," BioMed Research International, vol. 2019, 2019.

[13] W. Nimlamool, R. M. Andrews, and M. M. Falk, "Connexin43 phosphorylation by PKC and MAPK signals VEGF-mediated gap junction internalization," Molecular Biology of the Cell, vol. 26, no. 15, pp. 2755-2768, 2015.

[14] A. L. Harris, "Connexin channel permeability to cytoplasmic molecules," Progress in Biophysics and Molecular Biology, vol. 94, no. 1-2, pp. 120-143, 2007.

[15] H. Cai, B. Liu, T. Yang et al., "Involvement of PKCe in FSHinduced connexin 43 phosphorylation and oocyte maturation in mouse," Biology Open, vol. 7, no. 8, 2018.

[16] E. Winterhager and G. M. Kidder, "Gap junction connexins in female reproductive organs: implications for women's reproductive health," Human Reproduction Update, vol. 21, no. 3, pp. 340-352, 2015.

[17] C. L. Ackert, J. E. I. Gittens, M. J. O'Brien, J. J. Eppig, and G. M. Kidder, "Intercellular communication via connexin 43 gap junctions is required for ovarian folliculogenesis in the mouse," Developmental Biology, vol. 233, no. 2, pp. 258-270, 2001.

[18] R. Talhouk, C. Tarraf, L. Kobrossy et al., "Modulation of Cx43 and gap Junctional intercellular communication by androstenedione in rat polycystic ovary and granulosa cells in vitro," Journal of Reproduction \& Infertility, vol. 13, no. 1, pp. 21-32, 2012.

[19] M. Zhang, Y. Q. Su, K. Sugiura, G. Xia, and J. J. Eppig, “Granulosa cell ligand NPPC and its receptor NPR2 maintain meiotic arrest in mouse oocytes," Science, vol. 330, no. 6002, pp. 366-369, 2010.

[20] L. M. Mehlmann, "Stops and starts in mammalian oocytes: recent advances in understanding the regulation of meiotic arrest and oocyte maturation," Reproduction, vol. 130, no. 6, pp. 791-799, 2005. 
[21] Q. Liu, Y. Li, Y. Feng et al., "Single-cell analysis of differences in transcriptomic profiles of oocytes and cumulus cells at GV, MI, MII stages from PCOS patients," Scientific Reports, vol. 6, p. 39638, 2016.

[22] The Rotterdam ESHRE/ASRM-sponsored PCOS consensus workshop group, "Revised 2003 consensus on diagnostic criteria and long-term health risks related to polycystic ovary syndrome (PCOS)," Human Reproduction, vol. 19, no. 1, pp. 41-47, 2004.

[23] S. Picelli, O. R. Faridani, A. K. Bjorklund, G. Winberg, S. Sagasser, and R. Sandberg, "Full-length RNA-seq from single cells using smart-seq2," Nature Protocols, vol. 9, no. 1, pp. 171-181, 2014.

[24] G. M. Kidder and A. A. Mhawi, "Gap junctions and ovarian folliculogenesis,” Reproduction, vol. 123, no. 5, pp. 613-620, 2002.

[25] E. Gershon, V. Plaks, and N. Dekel, "Gap junctions in the ovary: expression, localization and function," Molecular and Cellular Endocrinology, vol. 282, no. 1-2, pp. 18-25, 2008.

[26] X. Zhao, F. du, X. Liu et al., "Brain-derived neurotrophic factor (BDNF) is expressed in buffalo (Bubalus bubalis) ovarian follicles and promotes oocyte maturation and early embryonic development," Theriogenology, vol. 130, pp. 79-88, 2019.

[27] H. Zhang, Q. Wei, Z. Gao et al., "G protein-coupled receptor 30 mediates meiosis resumption and gap junction communications downregulation in goat cumulus-oocyte complexes by $17 \beta$-estradiol," The Journal of Steroid Biochemistry and Molecular Biology, vol. 187, pp. 58-67, 2019.

[28] C. M. H. Combelles, M. J. Carabatsos, T. R. Kumar, M. M. Matzuk, and D. F. Albertini, "Hormonal control of somatic cell oocyte interactions during ovarian follicle development," Molecular Reproduction and Development, vol. 69, no. 3, pp. 347-355, 2004.

[29] H. E. Corbett, C. D. Dubé, S. Slow, M. Lever, J. M. Trasler, and J. M. Baltz, "Uptake of betaine into mouse cumulus-oocyte complexes via the SLC7A6 isoform of $\mathrm{y}+\mathrm{L}$ transporter," Biology of Reproduction, vol. 90, no. 4, p. 81, 2014.

[30] J. E. Gittens and G. M. Kidder, "Differential contributions of connexin 37 and connexin 43 to oogenesis revealed in chimeric reaggregated mouse ovaries," Journal of Cell Science, vol. 118, pp. 5071-5078, 2005.

[31] G. FitzHarris and J. M. Baltz, "Regulation of intracellular $\mathrm{pH}$ during oocyte growth and maturation in mammals," Reproduction, vol. 138, no. 4, pp. 619-627, 2009.

[32] V. Lodde, F. Franciosi, I. Tessaro, S. C. Modina, and A. M. Luciano, "Role of gap junction-mediated communications in regulating large-scale chromatin configuration remodeling and embryonic developmental competence acquisition in fully grown bovine oocyte," Journal of Assisted Reproduction and Genetics, vol. 30, no. 9, pp. 1219-1226, 2013.

[33] M. R. Maduro, "The signaling pathways guiding PCOS," Reproductive Sciences, vol. 25, no. 7, pp. 965-966, 2018.

[34] J. Y. Park, Y. Q. Su, M. Ariga, E. Law, S. L. Jin, and M. Conti, "EGF-like growth factors as mediators of LH action in the ovulatory follicle," Science, vol. 303, no. 5658, pp. 682-684, 2004.
[35] R. Prochazka, M. Blaha, and L. Němcová, "Significance of epidermal growth factor receptor signaling for acquisition of meiotic and developmental competence in mammalian oocytes," Biology of Reproduction, vol. 97, no. 4, pp. 537-549, 2017.

[36] T. Shinohara, Y. Ohta, N. Kawate et al., "Treatment with the MAPK kinase inhibitor U0126 during the first two hours of in vitro maturation improves bovine oocyte developmental competence," Reproduction in Domestic Animals, vol. 53, no. 1, pp. 270-273, 2018. 